\title{
Nanotubos de Carbono - Moldes para Cristais Unidimensionais
}

\author{
PEDROM.F.J. COSTA, MALCOLM L.H. GREEN
}

\begin{abstract}
Nano é definitivamente a palavra do momento. Não só comporta novos e excitantes desenvolvimentos de materiais e estruturas, mas também engloba toda uma área inexplorada de ciência fundamental onde a mecânica quântica se funde com a física clássica. Entre as descobertas mais promissoras da nanotecnologia encontram-se os nanotubos de carbono. Num intervalo de menos de 10 anos, as notáveis propriedades eléctricas e mecânicas dos nanotubos de carbono têm capturado a atenção de investigadores por todo o mundo. Tal deve-se, em grande parte, ao facto destas novas estruturas serem de interesse fundamental e poderem, eventualmente, conduzir a um leque de aplicações gigantesco com um potencial económico avaliado em muitos milhões de euros. As referidas aplicações vão desde a electrónica em nanoescala (nanoelectrónica) e instrumentos para manipular moléculas individualmente, até materiais excepcionalmente resistentes, passando pelos monitores de ecrã plano e células de combustivel de hidrogénio.
\end{abstract}

\section{Introdução}

O conceito de nanotecnologia teve um enorme impulso com uma das comunicações de Richard Feynman, em Dezembro de 1959, "There's plenty of room at the bottom"[1]. As seguintes palavras, enunciadas no encontro da Sociedade Americana de Física no Caltech, foram um momento único e determinante na nanoaventura:
"What I want to talk about is the problem of manipulating and controlling things on a small scale[...] What I have demonstrated is that there is room - that you can decrease the size of things in a practical way. I now want to show that there is plenty of room. I will not now discuss how we are going to do it but only what is possible in principle - in other words, what is possible according to the laws of physics[...] We are not doing it simply because we haven't yet gotten around to it."

Presentemente existem programas científicos que foram ou estão a ser erigidos para promover a nanotecnologia ao redor do globo. Vários governos têm investido em iniciativas destinadas a transformar o nanomundo numa realidade para o grande público no mais breve trecho de tempo. Esta tecnologia vai provavelmente induzir, nas próximas duas décadas, uma série de alterações, e mesmo revoluções industriais, que mudarão por completo as nossas vidas, muito para além do que a microelectrónica de silício fez no século XX. Desde espantosas aplicações biomédicas, de computadores quânticos e materiais feitos por medida, quase tudo sentirá os efeitos da nanorevolução. Nesta instância, os nanotubos de carbono poderão desempenhar um papel crucial caso as suas fantásticas propriedades eléctricas e mecânicas possam ser plenamente exploradas.

A realidade dos fulerenos surgiu em 1985 com uma colaboração de Harry Kroto, da Universidade de Sussex, UK, e Richard Smalley, da Universidade Rice,
USA, quando investigavam as propriedades do carbono presente no espaço interestelar [2]. A descoberta da estrutura de carbono em forma de bola $\mathrm{C}_{60}$, à qual chamaram Buckminsterfulereno, tomou a comunidade científica de surpresa e iniciou imediatamente uma nova era da química de carbono. Foi, todavia, necessário esperar até 1990 para uma equipa liderada por Wolfgang Kratschmer, no Instituto Max Planck para a Física Nuclear, em Heidelberg, Alemanha, e Donald Huffman, da Universidade do Arizona, USA, conseguir a produção em massa de fulerenos por meio de uma corrente eléctrica transmitida entre dois eléctrodos de grafite em atmosfera de hélio [3].

Em 1991, nos laboratórios da NEC, Japão, Sumio lijima usou um método similar, mas deixou os eléctrodos separados por uma pequena distância. Com este procedimento conseguiu fazer crescer umas finas fibras de carbono no eléctrodo negativo [4]. lijima estudou os microtúbulos usando Microscopia Electrónica de Transmissão de Alta Resolução (HRTEM) e mapas de Difracção Electrónica (ED). Estes primeiros tubos eram, na verdade, Nanotubos de Carbono de Parede Múltipla (MWNTs). lijima verificou serem estruturas com camadas de grafite enroladas na forma de cilindros e acomodadas umas dentro das outras, à semelhança das famosas bonecas russas. Pouco depois desta descoberta observou-se que a adição de um catalizador metálico levava à síntese de nanotubos com uma única camada de carbono grafitico, os Nanotubos de 
Carbono de Parede Única (SWNTs) [5]. Os nanotubos de carbono não devem ser confundidos com as fibras de carbono que são comercialmente produzidas desde há algumas décadas. As fibras de carbono têm uma variedade de diâmetros, geralmente acima dos $50 \mathrm{~nm}$, conforme o método de produção e podem apresentar vários graus de ordem estrutural sendo constituídas por planos grafiticos paralelos ao eixo vazio da fibra.

Alguns investigadores afirmam que lijima não foi o primeiro a descobrir os nanotubos de carbono. De facto, em 1960, Roger Bacon da Companhia Nacional de Carbono em Ohio, produziu e caracterizou microrolos de grafite [6]. Posteriormente, em 1979, na $14^{\text {a }}$ Conferência Bienal de Carbono, Peter Willes e John Abrahanmson apresentaram os resultados de uma pesquisa na qual fibras de carbono foram obtidas a partir de uma descarga eléctrica entre dois eléctrodos de grafite. Os aglomerados de pequenas fibras foram caracterizados por difracção electrónica [7].

Não obstante a controvérsia, o trabalho de lijima despoletou um campo de investigação completamente novo, pleno de oportunidades em química. É impressionante observar o enorme trabaIho realizado desde 1991, traduzido na quantidade de artigos publicados.

Têm sido atribuídas aos nanotubos diversas propriedades espantosas - alta estabilidade térmica, enorme resistência à tracção e uma superlativa resiliência pelo que projectos fantásticos de robots atómicos (nanobots), edificios resistentes a sismos e mesmo elevadores capazes de nos transportar ao espaço têm sido imaginados. Os nanotubos são, indubitavelmente, um dos materiais mais resistentes que a humanidade conhece. Com uma resistência à tracção cem vezes superior à do aço e com um módulo de Young de 1.2 TPa toleram também uma enorme tensão antes de qualquer falha mecânica [8]. Crê-se, no entanto, que no futuro próximo a mais rentável das aplicações para os nanotubos será no domínio electrónico. Na realidade, uma das características marcantes destas estruturas é a capacidade de conduzirem electricidade ou exibirem comportamento semicondutor dependendo apenas de factores puramente geométricos tais como o diâmetro e a quiralidade dos nanotubos.

Previsivelmente, a continua miniaturização dos microprocessadores vai obrigar os fabricantes de computadores a procurar substitutos da sílica uma vez que o processo e materiais hoje usados vão rapidamente atingir os seus limites físicos. Os nanotubos de carbono são, certamente, fortes candidatos para desempenhar este papel.

Mas nem tudo são promessas. Assim, os nanotubos de carbono revelaram já a sua utilidade para vários fins que abarcam desde a significante melhoria de microscópicos de sonda de varrimento [9] até ao seu uso como nanopinças para agarrar e mover partículas [10], passando pela criação de nanotermó- metros [11] e circuitos lógicos para computadores [12].

\section{Estrutura e nomenclatura de nanotubos}

Até 1985, o diamante e a grafite eram as únicas duas fases cristalinas conhecidas de carbono sólido. O primeiro é um material extremamente forte no qual os quatro electrões de valência do carbono são igualmente partilhados originando um estado híbrido $\mathrm{sp}^{3}$ tetraedricamente coordenado, o qual explica a sua estrutura rígida. Por seu lado, a grafite consiste em camadas bidimensionais de átomos de carbono $\mathrm{sp}^{2}$ arranjadas numa rede hexagonal. Estas camadas apresentam extensa deslocalização da densidade electrónica tendo como consequência o comportamento semicondutor da grafite.

O novo grupo de compostos de carbono baseados numa estrutura de caixa fechada tem como exemplo mais conhecido o $\mathrm{C}_{60}$. Esta molécula tem uma estrutura de icosaedro truncado formada por 12 anéis pentagonais e 20 anéis hexagonais. A coordenação dos átomos de carbono nos fulerenos não é planar mas antes ligeiramente piramidal com algum carácter $\mathrm{sp}^{3}$ presente nos carbonos essencialmente $\mathrm{sp}^{2}$. Mas o ponto fulcral do $\mathrm{C}_{60}$ é a presença dos anéis pentagonais, os quais providenciam a curvatura necessária para formar a estrutura de caixa fechada. figura 1 Número de artigos publicados, organizados por ano, desde 1991 (fonte: CAS SciFinder Scholar, tópico de busca - "Carbon Nanotubes").

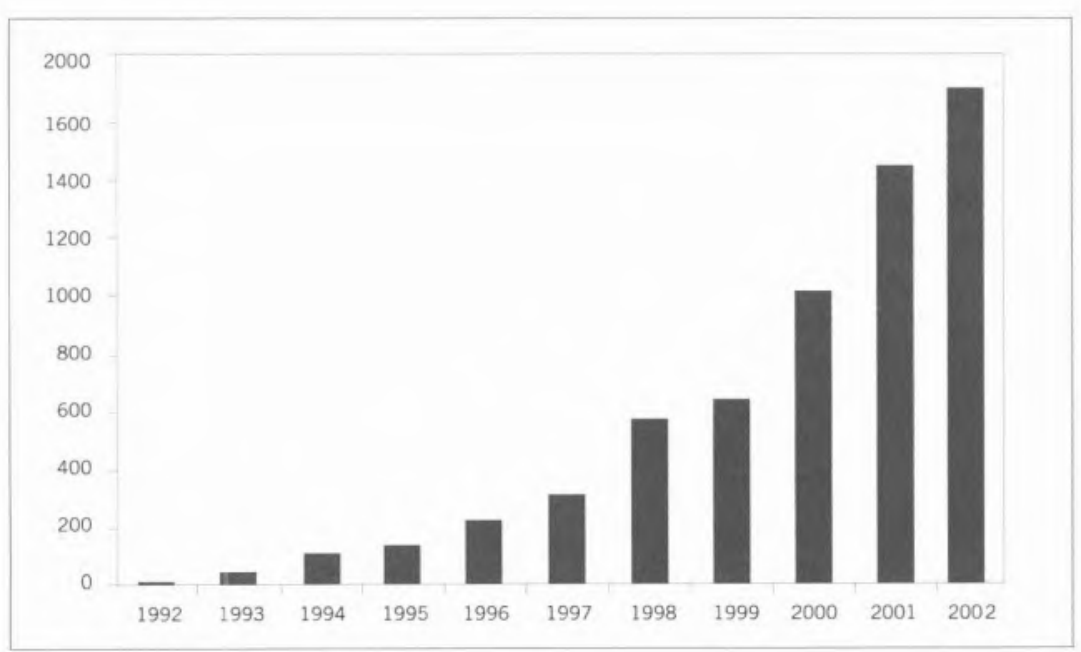




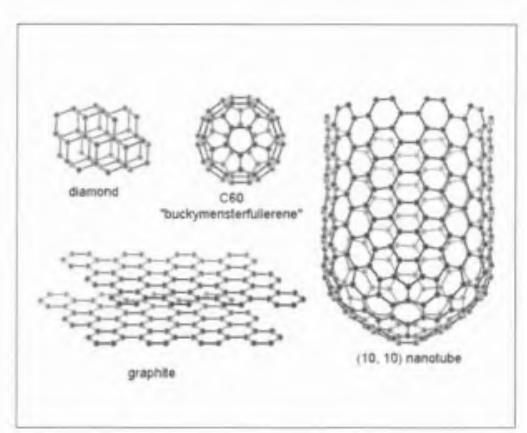

figura 2 Modelos de computador para os alótropos de carbono.

Com referência ao Teorema de Euler [13] podemos compreender como é possivel obter estas estrutura fechadas pela expressão que relaciona o número de vértices (V), arestas (E) e faces (F) de um objecto, $\mathrm{V}-\mathrm{E}+\mathrm{F}=\mathrm{X}$, onde $\mathrm{X}=2(1-\mathrm{g})$. $X$ é denominado a característica de Euler e está relacionado com o número de cavidades, g. Para uma esfera como - $\mathrm{C}_{60}, \mathrm{~g}=0$ enquanto que para um toro, $\mathrm{g}=1$. Para a rede hexagonal de grafite os carbonos $\mathrm{sp}^{2}$ implicam que $3 \mathrm{~V}=2 \mathrm{E}$ e pode ser derivada uma formulação mais útil do teorema,

$$
\ldots 2 n_{4}+n_{5}-n_{7}-2 n_{8} \ldots=\Sigma(6-x) n_{*}=12(1-g)
$$

onde $n_{x}$ é o numero de anéis com $x$ lados. Esta forma é muito útil na medida em que nos mostra como os anéis, sem ser hexágonos, deformam uma estrutu- ra a priori hexagonal plana e quantos serão necessários para provocar o encerramento da estrutura. Assim, se $\mathrm{g}=0$ e os pentágonos forem os únicos anéis não hexagonais serão necessários 12 pentágonos para fechar a rede hexagonal. Cada pentágono, por conseguinte, produz uma curvatura de $720 / 12=$ 60. Se um heptágono estiver presente, serão precisos 13 pentágonos para o encerramento. 0 heptágono induz, portanto, uma curvatura de -60 razão pela qual será necessário um pentágono extra.

Os nanotubos de carbono, por vezes também designados por fios quânticos, podem, como foi dito, ser de parede múltipla (MWNTs) ou de parede única (SWNTS). Os MWNTs consistem em uma série de cilindros concêntricos e fechados separados por ca. $3.45 \AA$, o que é ligeiramente maior que o espaçamento intercamada da grafite. Isto deve-se ao facto do número de carbonos aumentar à medida que passamos de um cilindro para outro mais externo sendo, consequentemente, impossivel manter um empilhamento $A B A B$ perfeito, como na grafite. Temos pois, para os MWNTs, um espaçamento intercamada próximo daquele da grafite turboestratificada. Nos SWNTs existe apenas um único tubo sem outras camadas de grafite que 0 envolvam. Um SWNT pode, portanto, ser visualizado cortando um $\mathrm{C}_{60}$ em dois e espaçando as duas metades com um cilindro de grafite com o mesmo diâmetro.

Durante o processo de enrolamento da camada de grafite pode-se introduzir helicidade pelo que os nanotubos são classificados como em zigzag, em cadeira ou quirais. Desta maneira, se $0 \mathrm{C}_{60}$ for bissectado normalmente a um eixo de grau 5 teremos um tubo em cadeira; no caso de ser bissectado normalmente a um eixo de grau 3 então o tubo será em zigzag. Os tubos em cadeira e zigzag são não quirais e não apresentam helicidade. Para além destes existe uma variedade de tubos quirais que apresentam um eixo em parafuso ao longo do eixo do tubo - a formação ocorre como no crescimento em espiral dos cristais. Os muitos diâmetros e helicidades dos nanotubos obrigaram ao desenvolvimento de um sistema simples de nomenclatura para identificar uma determinada geometria imediatamente. Os nanotubos de carbono têm, por esta razão, sido classificados de acordo com o diâmetro do tubo, $\mathrm{d}_{\mathrm{t}}$ e o ângulo quiral, $\theta$ [13]. Estas variáveis são mais bem ilustradas recorrendo a um diagrama da célula unitária do nanotubo de carbono. $\mathrm{O}$ chamado vector quiral, $\mathrm{C}_{\mathrm{n}}$, é definido pelos números inteiros $(n, m)$ e pelos vectores unitários $a_{1}$ e $a_{2}$ da malha hexagonal.

figura 3 Diagrama de um nanotubo $(4,2)$. 0 vector quiral $\mathrm{OA}\left(\mathrm{ou} \mathrm{C}_{h}\right.$ ) é definido na malha hexagonal pelos vectores unitários $a_{1}$ e $a_{2}$ e o ângulo quiral $\theta$ é definido em relação ao eixo de zigzag (adaptado de [13]). 
Portanto, um tubo é formado enrolando uma camada de grafite de tal forma que os dois pontos ligados pelo vector quiral, $\mathrm{C}_{\mathrm{h}}$, coincidam. Muitos pares $(\mathrm{n}, \mathrm{m})$ podem ser ilustrados definindo cada um uma forma diferente de enrolar a camada de grafite para formar um nanotubo de carbono como uma quiralidade definida. Os casos limites são $n=0, m=0$ (tubo em zigzag) e $n=m=0$ (tubo em cadeira). Para um nanotubo de carbono definido pelos índices $(n, m)$, o diâmetro, dt e 0 ângulo quiral $\theta$ são dados por.

$$
d_{t}=a \frac{\sqrt{m^{2}+m n+n^{2}}}{\pi} \quad a=1.42 \sqrt{3}
$$

$$
\theta=\arctan \left(\frac{\sqrt{3} m}{2 n+m}\right) \quad 0 \leq \theta \leq 30^{\circ}
$$

\section{Síntese de nanotubos de carbono}

Muitas têm sido as técnicas usadas para sintetizar nanotubos. Os últimos anos trouxeram métodos mais eficientes e é hoje razoável pensar na produção de $\mathrm{kg} /$ dia de nanotubos. Todavia, as principais formas de sintese permanecem a técnica Kratschmer-Huffman de arco eléctrico, a pirólise catalítica de gases e a ablação laser, com a segunda a posicionar-se como a mais promissora das três.

0 primeiro método que permitiu a produção de quantidades isoláveis de fulerenos e nanotubos foi a descarga de arco. Esta envolve a vaporização de um ânodo de grafite por um arco eléctrico dentro de uma câmara que usualmente contém uma pressão reduzida de gás ( $\mathrm{He}, \mathrm{Ar}, \mathrm{H}_{2}$ ou outro). A utilização de um eléctrodo de grafite pura resulta na formação de um depósito duro no cátodo contendo o seu núcleo macio os MWNTs. Estes têm comprimentos de mais de $10 \mathrm{~mm}$ e diâmetros na casa dos 5 - $30 \mathrm{~nm}$. Juntamente é também produzida uma camada de fuligem de carbono que se deposita nas paredes da câmara. Esta fuligem contém uma variedade de fulerenos que podem ser ex- traídos com alto grau de pureza através de uma lavagem com tolueno e extracção Soxhlet.

De forma a obter SWNTs a partir deste processo, o eléctrodo de grafite deve ser cheio com uma mistura de catalizadores metálicos. Algumas sínteses bem sucedidas têm usado metais dopantes que incluem entre outros, compósitos de $\mathrm{Cu}$ [14], os metais do grupo da platina (Rh, Pd, Pt) [15] ou Gd e La [16]. No entanto, os catalizadores metálicos com maior sucesso são o Fe, Co e Ni [17]. Varias sinteses descritas na literatura empregaram misturas dos metais acima descritos colocados na cavidade de um eléctrodo de grafite oco. De facto, o meIhor rendimento obtido para a síntese de SWNTs usando a técnica de arco faz-se por meio de uma mistura de Ni/Y/grafite na razão 4.2:1:92.8 (\%m), respectivamente [18]. Os SWNTs podem também ser preparados usando uma variedade de óxidos $\left(\mathrm{Y}_{2} \mathrm{O}_{3}, \mathrm{LaO}_{3}, \mathrm{CeO}_{2}\right)$ como catalizadores [16, 19]. Estes nanotubos podem ocorrer num pequeno "colar" em redor da ponta do cátodo, como um material género teia-de-aranha depositado atrás do cátodo ou na fuligem condensada nas paredes da câmara. A maior densidade de tubos encontra-se localizada no "colar". O diâmetro médio dos SWNTs é de aproximadamente $1.4 \mathrm{~nm}$ e o seu comprimento pode ir até $10 \mathrm{~mm}$, estando usualmente ligados em feixes por interacçōes de van der Waals.

Para além da técnica convencional de arco, os nanotubos de carbono são produzidos por decomposição de hidrocarbonetos. Tal envolve a passagem de um gás contendo carbono (usualmente $\mathrm{CO}$, metano, etano, acetileno, ...) sobre um catalizador na forma de pó ou disperso numa placa, dentro de um forno entre $500-1000{ }^{\circ} \mathrm{C}$. Um método alternativo envolve a vaporização do catalizador no fluxo de gás que atravessa o forno a alta temperatura (método do catalizador flutuante). O ferroceno ou outro composto metálico facilmente sublimável são normalmente usados. A espécie catalizadora é vaporizada para dentro do fluxo de gás, decompondo-se a alta temperatura para formar pequenos clusters em fase gasosa. De facto, os métodos desenvol-

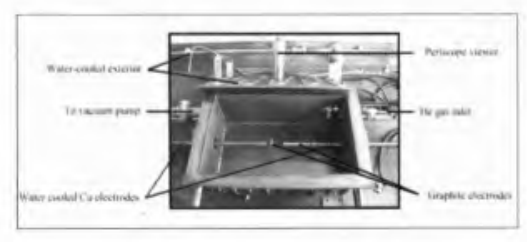

figura 4 Um reactor Kratschmer-Huffiman manualmente controlado.

vidos para a pirólise catalitica assemeIham-se bastante aos da produção das fibras de carbono. Na técnica de deposição em placa, o catalizador é preparado na forma de pequenos agregados de diâmetro e espessura desejados recorrendo à Deposição Química de Vapor (CVD) ou pela deposição de metal a partir de suspensões coloidais controladas. Os metais usados são semelhantes aos da síntese de arco eléctrico. Estes métodos de sintese têm o benefício de originarem menos carbono grafitico no produto final.

Relativamente ao mecanismo de formação, crê-se que a elevadas temperaturas a superficie da partícula metálica catalisa a decomposição do hidrocarboneto em carbono e $\mathrm{H}_{2}$. $\mathrm{O}$ carbono é então adsorvido e difunde-se para o interior da partícula como carboneto metálico sendo extrudido do lado oposto na forma de nanotubo. Este processo continuará até que a fonte de carbono seja removida ou a partícula metálica envenenada. A presença de agregados de metais de transição é essencial para a formação de nanotubos no processo de pirólise sendo o diâmetro do nanotubo determinado pelo tamanho da partícula metálica.

A pirólise catalítica tem-se revelado essencial na sintese de nanotubos alinhados, necessários para a fabricação de ecrãs planos. 0 alinhamento é obtido por CVD sobre metais de transição embebidos nos poros de sílica mesoporosa ou nos canais de membranas de alumina. Rao et al., por exemplo, afirmam que o comprimento médio dos nanotubos pode ser de $60 \mathrm{~mm}$ usando metano e acetileno como gases. No seu trabaIho, uma proporção negligenciável de nanopartículas metálicas cobertas de grafite foi observada ao longo dos nanotubos alinhados [20]. 
figura 5 (A) Imagem de fase mostrando a projecçẫo $\langle 110\rangle$ do KI incorporado dentro de um SWNT com $1.6 \mathrm{~nm}$ de diâmetro, reconstruida a partir de uma série focal de 20 imagens. As frequências espaciais máxima e mínima de $1 /(0.23 \mathrm{~nm})$ e 1/(1.05 $\mathrm{nm})$. respectivamente, foram retidas com um filtro de Wiener. No canto superior esquerdo a imagem mostra um aumento da região 1 e uma illustraçâo esquemática representando arranjos alternados de camadas $\{100)$ I-2K-3I$2 \mathrm{~K}-\mathrm{I}$ e K-2I-3K-2I-K dentro do cristal. Ainda $\mathrm{em}$ (A) no canto inferior direito encontra-se o modelo de superficie da região. (B a F) Perfis de linha de pixel único obtidos a partir das linhas marcadas de $\mathrm{B}$ a $\mathrm{F}$ no canto superior esquerdo de (A). O nivel de fundo destes perfis é arbitrário porque o procedimento de

reconstrução não recupera variações de baixa frequência espacial em fase. Estruturas esquemáticas do cristal mostrando os átomos que contribuem para o contraste sâo também representadas (adaptado de [23]).

Ainda relativo à técnica de CVD também têm sido usados zeólitos contendo canais unidimensionais na síntese de SWNTs isolados [21].

O terceiro método acima referido, a ablação por laser, foi referido pela primeira vez em 1995 [22]. Nesta técnica, optimizada na Universidade de Rice, USA, um laser é usado para vaporizar um alvo de carbono aquecido e previamente impregnado com um catalizador metálico, normalmente níquel, cobalto, ferro, ou misturas destes metais. $\mathrm{O}$ alvo é colocado num tubo de quartzo em atmosfera de hélio, dentro de um forno a cerca de $1200^{\circ} \mathrm{C}$ e rodado de forma a maximizar o rendimento total, enquanto se processa a vaporização. 0 produto condensado é arrastado para um colector arrefecido pelo fluxo de um gás inerte. Crê-se que o crescimento dos nanotubos ocorre na fase gasosa à medida que o alvo é evaporado. Com este método obtêm-se enormes feixes de SWNTs com rendimentos de cerca de $80 \%$. Os restantes produtos são fulerenos e nanopartículas grafiticas, facilmente removíveis. Estes SWNTs são quase uniformes em diâmetro apresentando-se numa malha $2 \mathrm{D}$ triangular de 100 a 500 tubos. A ablação por laser possibilita, portanto, a produção de SWNTs de alta qualidade numa escala de $1-10 \mathrm{~g}$.

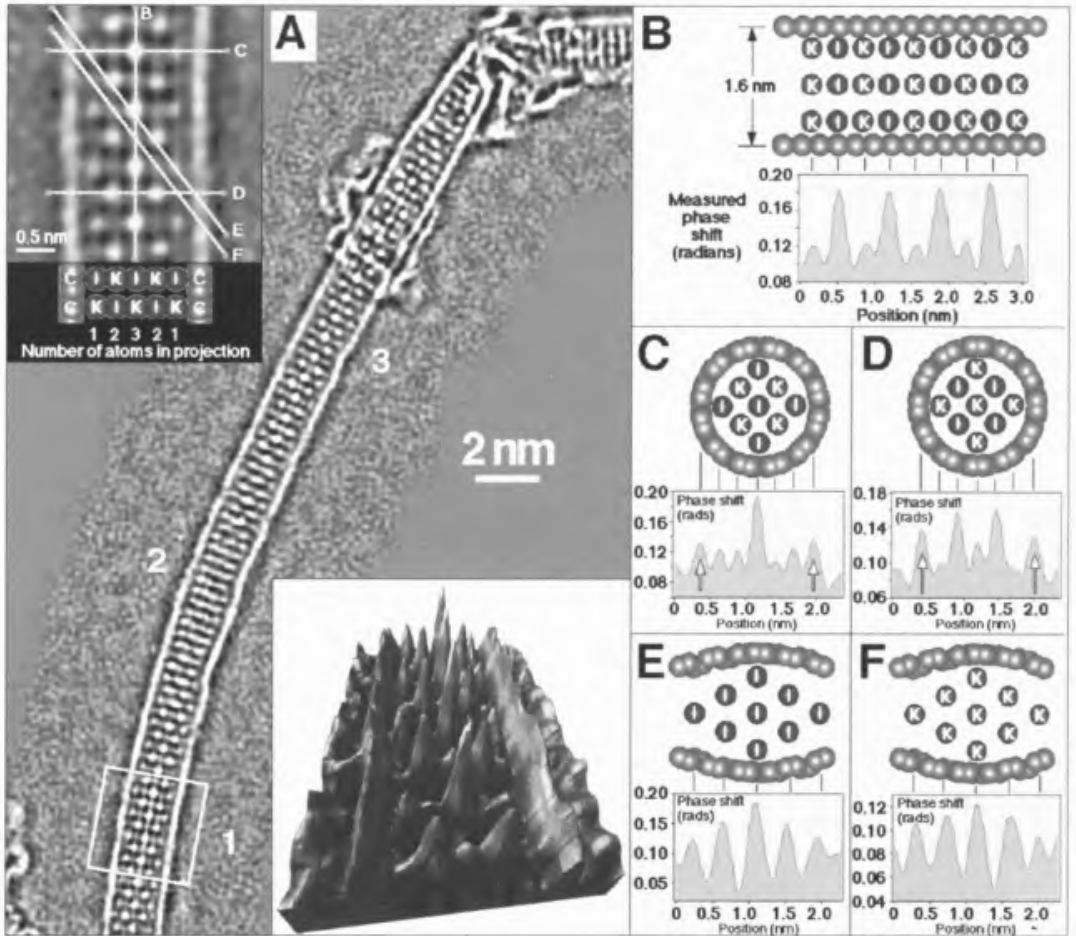

\section{Caracterização de nanotubos}

A descoberta dos fulerenos deve-se, em grande parte, à observação dos seus picos característicos no espectro de massa de carbono vaporizado por laser. 0 modelo de bola de futebol foi proposto de forma a explicar a predominância do $\mathrm{C}_{60}$. Entretanto, com a descoberta dos fulerenos tubulares, outras técnicas de caracterização têm sido empregues. Entre estas, as mais comuns e bem sucedidas têm sido, indubitavelmente, as técnicas nanoscópicas capazes de visualizar materiais com uma resolução no intervalo de $<0.1$ a $10 \mathrm{~nm}$ que englobam a Microscopia Electrónica de Transmissão de Alta Resolução (HRTEM), a Microscopia Electrónica de Varrimento de Transmissão (STEM), a Microscopia Electrónica de Varrimento (SEM) a as técnicas de sondas de varrimento (SPMS), como a Microscopia de Força Atómica (AFM) e o Microscopia de Varrimento de Túnel (STM). Enquanto muitas destas técnicas são apreciadas intrinsecamente pelas suas propriedades de visualização a maioria apresenta também a capacidade de combinar características analíticas adicionais no mesmo instrumento. O TEM tem sido particularmente bem sucedido neste campo e técnicas como a espectroscopia de Energia Dispersiva de
Raios-X (EDX) ou a Espectroscopia de Perda de Energia Electrónica (EELS) podem ser agora combinadas no mesmo aparelho.

O HRTEM foi o primeiro método a ser aplicado com vista a caracterização de MWNTs [4]. No seu importante estudo, lijima foi capaz, não só de caracterizar os MWNTs em termos da sua natureza tubular concêntrica, mas também, por análise detalhada de padrões de Difracção Electrónica (ED), de determinar a sua natureza helicoidal. 0 mesmo autor foi também responsável pela primeira imagem de SWNTs [5]. A espectroscopia EDX pode ser usada para analisar os elementos presentes numa pequena área da amostra, seja a análise de partículas de catalizador ou a determinação dos componentes presentes no interior de um nanotubo preenchido. Ainda relativas à microscopia de transmissão, outras técnicas correntemente utilizadas incluem a reconstrução de imagens, na qual uma série de imagens de HRTEM é recombinada computacionalmente, eliminando aberrações, resultando num aumento significativo da resolução [23].

Devido ao tamanho da área observada por TEM (na escala dos micrómetros) este método de análise não é representativo de toda a amostra. Desta forma, outra técnica microscópica que tem 


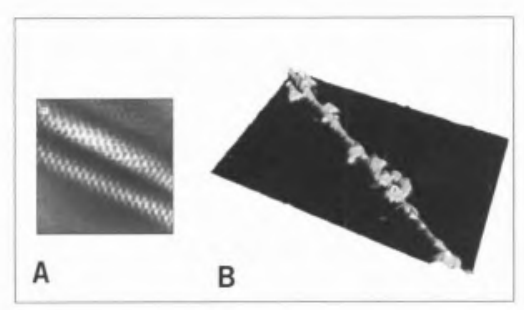

figura $6 \mathrm{~A}$ - Imagem STM da estrutura atómica de um SWNT isolado num substrato Au(111) (adaptado de [24]); B- Representação topográfica $3 \mathrm{D}$ realizada com $A F M$ de um SWNT com nanopartículas de Au covalentemente ligadas (tirada de [25]).

sido largamente usada é o SEM. Na generalidade dos SEMS presentemente disponiveis podemos facilmente obter uma boa resolução para ampliações até 30 - 40 k, dependendo a resolução maioritariamente do tipo de material e da morfologia da superfície. SEMs de alta resolução (FEG-SEM) podem alcançar ampliações de 100 k. Pelo facto de serem fáceis de manusear e de preparar amostras para visualização, esta técnica constitui um método rápido e fiável para verificar rendimentos ou julgar graus de pureza e o alinhamento de nanotubos.

Os métodos de sonda de varrimento tais como AFM e STM podem produzir imagens de ainda maior ampliação que as obtidas com HRTEM, com resoluções próximas de $0.1 \mathrm{~nm}$. Estas técnicas têm permitido a visualização directa da estrutura atómica externa dos nanotubos de carbono. Por exemplo, o carácter helicoidal dos MWNTs e SWNTs foi confirmado, sem ambiguidades, recorrendo

figura 7 Espectro Raman de uma amostra de SWNTs produzidos por descarga de arco (laser ao AFM e STM. Para além de sondarem a ordem atómica local dos SWNTs, os SPMs podem também detectar comportamentos derivados de defeitos nos nanotubos e adicionalmente, quaisquer outras modificaçōes na superfície dos mesmos. O STM tem, por exemplo, sido usado para analisar defeitos estruturais $\mathrm{sp}^{3}$, encerramento das pontas dos tubos e outras alterações induzidas por pentágonos. É igualmente possível determinar a quiralidade dos nanotubos a qual, como anteriormente se referiu, influencia as propriedades electrónicas dos nanotubos.

A espectroscopia Raman tem também proporcionado importantes informaçōes sobre a estrutura dos nanotubos. A frequência dos modos de Raman permitidos depende do diâmetro do tubo e do ângulo quiral, sendo o número de modos independente do diâmetro.

A espectroscopia Raman é um método satisfatório para verificar a qualidade de uma amostra uma vez que nos permite comparar a abundância de SWNTS com a quantidade de depósitos carbonáceos. A quantidade relativa destes tipos de materiais de carbono é rapidamente obtida a partir do espectro, integrando as bandas especificas dos SWNTs e comparando-as com a intensidade integrada total do espectro.

Um espectro de Raman dos nanotubos de carbono tem certas regiões distintas de identificação. Primeiro, existem ge- ralmente duas grandes bandas a comprimento de onda mais elevado, na região 1550 - $1600 \mathrm{~cm}^{-1}$ e que correspondem aos modos tangenciais de estiramento. A frequências mais baixas há uma série de picos devido aos modos de respiração radial dos SWNTS - 0 modo $A_{1 \mathrm{~g}}$ dos SWNTs aparece entre $100-250 \mathrm{~cm}^{-1}$ e pode ser usado para determinar o diâmetro dos SWNTs. A banda larga que aparece na região $2550-2700 \mathrm{~cm}^{-1}$ é geralmente interpretada como um sobretom de segunda ordem.

Uma outra técnica utilizada para determinar a pureza dos nanotubos é a análise termogravimétrica (TGA) na qual uma pequena amostra é colocada numa balança de precisão e aquecida. À medida que a temperatura aumenta a massa da amostra reduz-se. Os gráficos de perda de massa e de diferencial térmico versus temperatura podem ser facilmente traçados.

Também as medidas de Difracção de Raios-X (XRD) têm, por vezes, sido empregues na caracterização dos nanotubos de carbono. Os padrōes de XRD dos nanotubos mostram apenas as reflexões \{hk0\} e $\{001\}$ mas não as reflexões genéricas \{hkl\} das grafites turboestratificadas.

\section{Preenchimento de nanotubos}

Os nanotubos de carbono apresentam capilares internos com dimensões próxi-

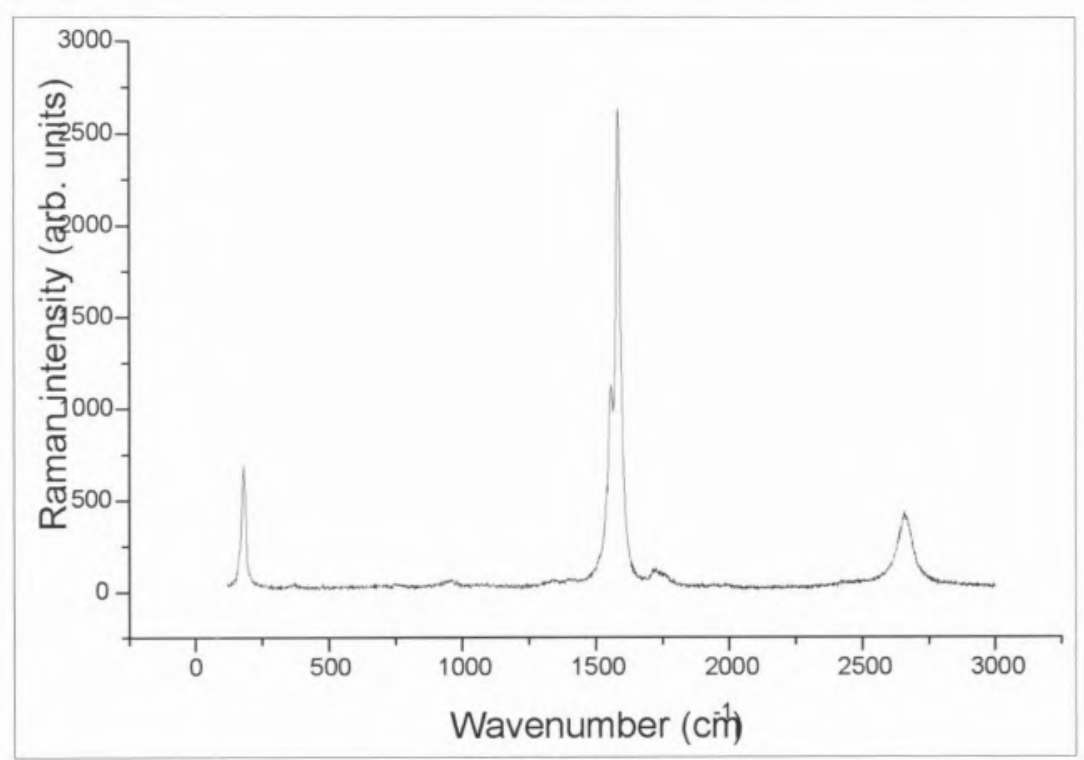




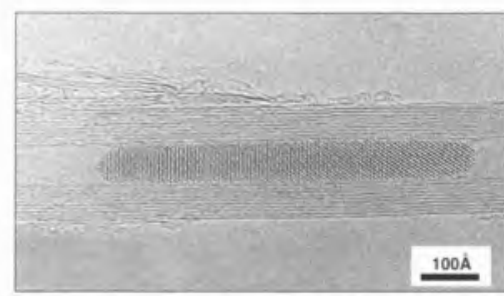

figura 8 Cristal encapsulado de $\mathrm{Sm}_{2} \mathrm{O}_{3}$ dentro de um MWNT (tirado de [32]).

mas dos limites de nucleação e crescimento cristalino de vários materiais. Neste ambiente restrito, esperar-se-ão alterações consideráveis das propriedades estruturais e físicas dos materiais incorporados. Estudos dedicados à química e física de incorporação em nanotubos podem conduzir tanto ao desenvolvimento de novos tipos de materiais hibridos como também a um entendimento mais lato do funcionamento dos materiais incorporados quando num ambiente monodimensional. Tem sido sugerido que os nanotubos preenchidos poder-se-iam utilizar como um meio eficiente para salvaguardar informação. A elevada razão comprimento/ diâmetro dos nanotubos é favorável ao seu uso como meio de suporte para catálise heterogénea, providenciando uma enorme área superficial para as reacções ocorrerem. Química in tubulo, onde a reacção ocorre no interior do nanotubo com a formação de um produto incapaz de escapar depois de sintetizado - seja a síntese de polímeros dentro de nanotubos - é tida como uma outra possível aplicação.

Ajayan foi o primeiro a observar que quando os MWNTs são aquecidos na presença de chumbo acima dos $400^{\circ} \mathrm{C}$ ao ar, as suas pontas ficam abertas e os tubos são preenchidos com o metal por via capilar [26]. Este trabalho conduziu à investigação de métodos químicos simples para abrir as pontas dos MWNTs, de forma a poder obter o seu preenchimento. Várias técnicas foram apresentadas, todas elas envolvendo tratamentos oxidativos dos tubos [27]. Em 1994, Tsang et al. obtiveram altos rendimentos de MWNTs abertos através do refluxo destes em $\mathrm{HNO}_{3}$ concentrado [28]. Crê-se que os métodos oxidativos atacam preferencialmente os anéis pentagonais presentes na ponta do tubo pois estes sofrem maior tensão e são, por conseguinte, mais reactivos.

Uma vez aberto, é necessário prever quais os materiais que poderão encher o nanotubo e por que meios será mais bem introduzido no capilar. As tentativas iniciais de preenchimento usavam geralmente métodos de lavagem em solução com graus variáveis de êxito [29]. Estes métodos com soluções líquidas proporcionavam algum preenchimento de nanotubos, quase sempre na forma de pequenas cristalites em vez do desejável enchimento contínuo do capilar. Num outro método usou-se um aditivo metálico nos eléctrodos de grafite durante a síntese de arco de MWNTs de forma a encapsular metais ou carbonetos metálicos [30]. Este provou ser apenas parcialmente bem sucedido resultando na formação de MWNTs de morfologia irregular. Trata-se, portanto, de um método menos controlável que os tratamentos pós síntese.

De longe, a técnica mais estudada e generalizada envolve o preenchimento por técnicas de meio fundido. Nestas circunstâncias, os primeiros trabalhos realizaram-se após a previsão teórica feita por Broughton e Pedersen que sugeriram que os nanotubos podiam ser preenchidos por capilaridade se a tensão superficial dos material fundido fosse menor que o patamar de 100-200 $\mathrm{mN} / \mathrm{m}$ [31]. Muitas experiências tem sido feitas com MWNTs originando uma enorme variedade de enchimentos com sais como o $\mathrm{UCl}_{4}$ e $\mathrm{MoO}_{3}$ [32].

Foi necessário esperar atẻ 1998 para o preenchimento de um SWNT ser relatado [33]. Sloan et al. fizeram uma deposição de $\mathrm{RuCl}_{3}$ em soluçẫo dentro de um SWNT seguido por uma redução com $\mathrm{H}_{2}$. Este processo resultou num baixo rendimento de enchimento e na formação de pequenas cristalites de ruténio metálico. Desde entâo vários sistemas de metais halogenados têm sido sintetizados [34]. O método segue o mesmo procedimento do preenchimento dos MWNTs, sendo primeiro feita a análise dos diagramas de fase e tensão superficial dos sais ou misturas de sais metálicos que poderão encher os tubos. Estes são seguidamente misturados com os SWNTs e selados numa ampola de quartzo evacuada. A ampola é depois colocada num forno e lentamente aquecida a $20-50^{\circ} \mathrm{C}$ acima do ponto de fusão do sal. Finalmente deixa-se arrefecer. Quando analisados com HRTEM os produtos apresentam alto rendimento com enchimento contínuo dos SWNTS e boa cristalinidade.

Recentemente, a incorporação de $\mathrm{Bal}_{2}$ em SWNTs revelou uma estrutura de coordenação completamente diferente de qualquer outra conhecida para este sal [35]. Além disso, o recente enchimento de SWNTs com uma solução sólida metaestável unidimensional de $\mathrm{AgCl}_{1-x} \mathrm{I}_{x}$ [36] demonstrou que os nanotubos podem encapsular uma fantástica variedade de materiais possibilitando também a sua formação controlada.

\section{Perspectivas}

Os nanotubos de carbono apresentam uma vasta quantidade de oportunidades ao nível comercial e da ciência fundamental. São, sem dúvida, um dos mais excitantes e promissores materiais originados pela nanorevoluçâo. No entanto, existem ainda algumas questōes para resolver, entre as quais a necessidade de aperfeiçoamento das técnicas de síntese de maneira a que a produção de nanotubos possa ser realizada à razâo de toneladas/dia. Um outro desafio prende-se com a síntese de tubos com propriedades electrónicas previamente escolhidas. Em resumo, é essencial encontrar formas reprodutíveis para a preparaçâo de nanotubos com características desejadas. No entanto, o facto de nos possibilitarem a movimentação de nanopartículas, a futura realidade do seu uso em ecrãs planos (a Samsung já apresentou alguns protótipos), a possível utilização para armazenagem de hidrogénio ou como meio para reforçar materiais, tudo isto mais do que justifica a aposta no desenvolvimento da investigação neste nanodomínio.

\section{Abreviaturas}

As abreviaturas usadas encontram-se em inglês pelo que a lista seguinte serve de apoio. 
AFM - Atomic Force Microscopy;

CVD - Chemical Vapour Deposition;

ED - Electron Diffraction;

EDX - Energy Dispersive X-Ray Spec-

troscopy;

EELS - Electron Energy Loss Spectros-

copy;

FEG - Field Emission Gun;

HRTEM - High Resolution Transmission

Electron Microscopy;

MWNT - Multi Walled Carbon Nanotube;

SEM - Scanning Electron Microscopy;

SPM - Scanning Probe Microscopy;

STEM - Scanning Transmission Electron

Microscopy;

STM - Scanning Transmission Micros-

copy;

SWNT - Single Walled Carbon Nanotube;

TGA - Thermogravimetric Analysis;

XRD - X-Ray Diffraction.

\section{Leitura aconselhada}

Ajayan, P. Chemical Reviews 99, 1787 1799 (1999).

Physics World 13, 29 - 53 (2000).

Sloan, J., Kirkland, A., Hutchison, J., Green, M., Chemical Communications, (2002) 1319 -1332 .

Agradecimentos

Os autores gostariam de agradecer a Maria José Calhorda, Jeremy Sloan e Karl Coleman a leitura e comentário do manuscrito. Agradecemos também o Petroleum Research Fund, administrado pela American Chemical Society (Bolsa No. 33765-AC5), ao EPSRC (Bolsas Nos. GR/L59238 e GR/L22324) e a Colebrand Ltd pelo apoio financeiro. Pedro Costa agradece a Fundação para a Ciência e a Tecnologia

(SFRH/BD/3103/2000).

\section{Bibliografia}

[1] A copy of the lecture is available at www.its.caltech.edu/ feynman .

[2] Kroto, H., Heath, J., O'Brien, S., Curl, R., Smalley, R. Nature 318, (1985) 162 - 163.

[3] Kratschmer, W., Lamb, L., Fostiropoulos, K., Huffman, D. Nature 347, (1990) 354 357.

[4] lijima, S. Nature 354, (1991) 56 - 58.
[5] lijima, S., Ichihashi, T. Nature 363 , (1993) $603-605$.

[6] Bacon, R. Journal of Applied Physics 31. (1960) $283-290$.

[7] Abrahamson, J., Wiles, P., Rhoades, B. Abstract in proceedings of the 14 th Biennial Conference on Carbon, Pennsylvania State University, June 1979. Reprinted in Carbon 37. (1999) 1873-1874.

[8] Smalley, R., American Scientist 85 (1997) 324 - 337.

[9] Dai, H., Hafner, J., Rinzler, A., Colbert, D., Smalley, R. Nature 384, (1996) 147 150; Wong, S., Woolley, A., Joselevich, E., Cheung, C., Leiber, C. JACS 120, (1998) $8557-8558$.

[10] Kim, P., Lieber, C. Science 286, (1999) $2148-2150$.

[11] Gao, Y., Bando, Y. Nature 415, (2002) 599 .

[12] Tans, S., Verschueren, A., Dekker, C. Nature 393, (1998) 49 - 52; Huang, Y., Duan, X., Cui, Y., Lauhon, L., Kim, K., Lieber Science 294, (2001) 1314 - 1316; Bachtold, A., Hadley, P., Nakanishi, T., Dekker, C. Science 294, (2001) 1317- 1320.

[13] Dresselhaus, G., Dresselhaus, M. S., Saito, R. Physical Properties of Carbon Nanotubes, Imperial College Press (1998).

[14] Lin, X., Wang, X., Dravid, V., Chang, R., Ketterson, J. Applied Physics Letters 64 , (1994) $181-183$.

[15] Seraphin, S., Zhou, D., Jiao, J., Minke, M., Wang, S., Yadav, T., Withers, J. Chemical Physics Letters 217, (1994) 191 - 198;

Saito, Y., Tani, Y., Miyagawa, N., Mitsushima, Kasuya, A., Nishina, Y. Chemical Physics Letters 294, (1998) 593 - 598.

[16] Sait, Y., Kawabata, K., Okuda, M. Journal of Physical Chemistry 99, (1995) 16076 - 16079.

[17] Ajayan, P., Lambert, J., Bernier, P., Barbedette, L., Colliex, C., Planeix, J., Chemical Physics Letters 215, (1993) 509 - 517; Bethune, D., Kiang, C., Vries, M., Gorman, G., Savoy, R., Vasquez, J., Beyers, R. Nature 363, (1993) 605-607; Shi, Z., Lian, Y., Zhou, X., Gu, Z., Zhang, Y., lijima, S., Zhou, L., Yue, K., Zhang, S. Carbon 37, (1999) $1449-1453$.

[18] Journet, C., Maser, W., Bernier, P., Loiseau, A., Chapelle, M., Lefrant, S., Deniard, P., Lee, R., Fischer, J. Nature 388, (1997) $756-758$.

[19] Shi, Z., Zhou, X., Jin, Z., Gu, Z., Wang, J., Feng, S., Xu, X., Liu, Z. Solid State Communications 97, (1996) 371 - 375.
[20] Satishkumar, B., Govindaraj, A., Rao, C. Chemical Physics Letters 307, (1999) 158. 162.

[21] Wang, N., Li, G., Tang, Z. Chemical Physics Letters 339, (2001) 47 - 52.

[22] Guo, T., Nikolaev, P., Thess, A., Colbert, D., Smalley, R. Chemical Physics Letters 243, (1995) 49 - 54.

[23] Meyer, R., Sloan, J., Borkowski, R., Kirkland, A., Novotny, M., Bailey, S., Hutchison, J., Green, M. Science 289, (2000) 1324 1326.

[24] Odom, T., Huang, J., Kim, P., Lieber, C., Nature 391, (1998) 62 - 64 .

[25] Azamian, B., Coleman, K., Davis, J., Hanson, N., Green, M., Chemical Communications, (2002) $366-367$.

[26] Ajayan, P., lijima, S. Nature 361 , (1993) 333 - 334.

[27] Ajayan, P., Ebbesen, T., Ichihashi, T., lijima, S., Tanigaki, K., Hiura, H. Nature 362. (1993) 522 - 525; Tsang, S., Harris, P., Green, M. Nature 362, (1993) 520 - 522; Chen, Y., Green, M., Griffin, J., Hammer, J., Lago, R., Tsang, S. Advanced Materials 8 , (1996) 1012 - 1015.

[28] Tsang, S., Chen, Y., Harris, P., Green, M. Nature 372, (1994) 159 - 162.

[29] Chu, A., Cook, J., Heesom, J., Hutchison, J., Green, M., Sloan, J. Chemical Materials 8, (1996) 2751 - 2754.

[30] Loiseau, A., Pascard, H. Chemical Physics Letters 256, (1996) $246-252$.

[31] Pederson, M., Broughton, J. Physical Review Letters 69, (1992) 2689 - 2692.

[32] Sloan, J., Cook, J., Chu, A., Sibley, M., Green, M., Hutchinson, J. Journal of Solid State Chemistry 140, (1998) 83- 90; Chen, Y., Chu, A., Cook, J., Green, M., Harris, P., Heesom, R., Humphries, M., Sloan, J., Tsang, S., Turner, J. Journal of Materials Chemistry 7, (1997) 545- 549.

[33] Sloan, J., Hammer, J., Sibley, M., Green, M. Chemical Communications, (1998) 347 - 348.

[34] For a good review see Sloan, J., Friedrichs, S., Meyer, R., Kirkland, A., Hutchison, J., Green, M. Inorganica Chimica Acta 330, (2002) $1-12$.

[35] Sloan, J., Grosvenor, S., Friedrichs, S., Kirkland, A., Hutchinson, J., Green, M., Angewandte Chemie International Edition 114 (2002) 1204

[36] Sloan, J., Terrones, M., Nufer, S., Friederichs, S., Bailey, S., Woo, H., Ruhle, M., Hutchinson, J., Green, M., Journal of the American Chemical Society 124, (2002) 2116 - 2117. 